\title{
The first donation after circulatory death following withdrawal of life-sustaining treatment in Korea
}

\author{
Eunsil Jeong ${ }^{1}$, Jae-sook $\mathrm{Oh}^{2}$, Yong-min Lee ${ }^{2}$, Jae-myeong Lee ${ }^{3}$ \\ ${ }^{1}$ Transplantation Center, Korea University Anam Hospital, Seoul, Korea \\ ${ }^{2}$ Korea Organ and Tissue Donation Agency, Seoul, Korea \\ ${ }^{3}$ Department of Surgery, Korea University Anam Hospital, Seoul, Korea
}

Background: We would like to report the first case of successful organ donation after withdrawal of life-sustaining treatment (WLST) in Korea.

Methods: A 52-year-old male patient who had cerebral hemorrhage was the potential brain-dead donor. After passing the first brain death examination, according to the recommendation of a neurologist, electroencephalograms (EEG) were performed 5 times at intervals of 2 to 3 days and they did not show flat EEGs. Since the family members' willingness to donate organs was very strong, they agreed to donation after circulatory death (DCD) after WLST in the operating room (OR) without them. The patient was transferred to the OR at 7:30 PM on July 3,2020. Surgical drape was done for the donor. At 8:00 PM, an intensivist in charge of the patient performed extubation and stopping the vasopressors at the same time. In 1 minute, oxygen saturation $\left(\mathrm{SpO}_{2}\right)$ fell below 70\%, which meant functional warm ischemia time began. At 8:15 PM, asystole was confirmed, and after a 5-minute "no touch time", declaration of circulatory death was done by the intensivist at 8:20 PM. Afterwards, all recipient surgeons who were waiting with surgical gown at the next OR moved to the donor's OR and performed organ procurement surgery.

Results: Aortic clamp and HTK fluid perfusion started at 8:22 PM, 2 minutes after the incision started. Liver was out at 8:56 PM and kidney was out at 9:11 PM. Organs quality were good and they were donated well to the recipients.

Conclusions: Since this case started with donation after brain-death, it is strictly categorized as DCD IV. In DCD IV, if the life-sustaining treatment is stopped due to unsuccessful brain death determination, it becomes DCD III process. All potential recipients were already arranged through KONOS. We can actively perform DCD after WLST in Korea with the set-up of laws and systems for $D C D$. 